\title{
Partial Match Queries in Relaxed Multidimensional Search Trees ${ }^{1}$
}

\author{
C. Martínez, ${ }^{2}$ A. Panholzer, ${ }^{3}$ and H. Prodinger ${ }^{4}$
}

\begin{abstract}
Partial match queries arise frequently in the context of large databases, where each record contains a distinct multidimensional key, that is, the key of each record is a $K$-tuple of values. The components of a key are called the coordinates or attributes of the key. In a partial match query we specify the value of $s$ attributes, $0<s<K$, and leave the remaining $K-s$ attributes unspecified. The goal is to retrieve all the records in the database that match the specified attributes. In this paper we present several results about the average performance and variance of partial matches in relaxed $K$-dimensional trees (search trees and digital tries). These data structures are variants of the well known $K$ d-trees and $K$ d-tries. In relaxed trees the sequence of attributes used to guide a query is explicitly stored at the nodes of the tree and randomly generated and, in general, will be different for different search paths. In the standard variants, the sequence of attributes that guides a query examines the attributes in a cyclic fashion, fixed and identical for all search paths. We show that the probabilistic analysis of the relaxed multidimensional trees is very similar to that of standard $K d$-trees and $K$ d-tries, and also to the analysis of quadtrees. In fact, besides the average cost and variance of partial match in relaxed $K$ d-trees and $K$ d-tries, we also obtain the variance of partial matches in two-dimensional quadtrees. We also compute the average cost of partial matches in other relaxed multidimensional digital tries, namely, relaxed $K$ d-Patricia and relaxed $K$ d-digital search trees.
\end{abstract}

Key Words. Analysis of algorithms, Multidimensional search trees, $K$-dimensional binary search trees, Relaxed $K$-dimensional search trees, Quadtrees.

1. Introduction. In many applications such as Geographical Information Systems, databases, computer graphics, and statistical data analysis, the need frequently arises to maintain a set $F$ (also called a file) of multidimensional records dynamically [17]. Such a file should support insertions of new multidimensional records, deletions, and several associative queries, i.e., different types of operations that retrieve information from the file, given a condition on the keys of the records.

Each multidimensional record in the file contains a distinct multidimensional key and associated data. For the sake of simplicity, we will identify each record with its multidimensional key, which is an ordered $K$-tuple $\mathbf{x}=\left(x^{(0)}, x^{(1)}, \ldots, x^{(K-1)}\right)$. For $0 \leq j<K, x^{(j)}$ is the value of the $j$ th attribute (also the $j$ th coordinate) of $\mathbf{x}$. Each

\footnotetext{
${ }^{1}$ This research was supported by Acciones Integradas Hispano-Austríacas HU1997-0016 (Austrian-Spanish Scientific Exchange Program). The first author was also supported by ESPRIT LTR 20244 (ALCOM IT), CICYT TIC97-1475-CE, DGES PB95-0787 (KOALA), and CIRIT 1997SGR-00366 (SGR). The second author was also supported by the Austrian Research Society (FWF) under Project P12599-MAT.

2 Departament de Llenguatges i Sistemes Informàtics, Polytechnic University of Catalonia, Jordi Girona 1-3, E-08034 Barcelona, Spain. Conrado.Martinez@1si.upc.es.

${ }^{3}$ Institut für Algebra und Computermathematik, Technical University of Vienna, Wiedner Hauptstraße 8-10, A-1040 Vienna, Austria. Alois.Panholzer@tuwien.ac.at.

${ }^{4}$ Mathematics Department, University of Witwatersrand, P.O. Wits, 2050 Johannesburg, South Africa. helmut@gauss.cam.wits.ac.za.
}

Received September 15, 1998; revised March 16, 1999. Communicated by H. Prodinger and W. Szpankowski. Online publication October 13, 2000. 
$x^{(j)}$ belongs to some totally ordered domain $D_{j}$, and thus $\mathbf{x}$ is an element of $D=$ $D_{0} \times D_{1} \times \cdots \times D_{K-1}$. Therefore, each (multidimensional) key may be viewed as a point in a $K$-dimensional space. The analogy can be further exploited as we can, without loss of generality, assume that $D_{j}=[0,1]$ for all $0 \leq j<K$ and hence that $D$ is the hypercube $[0,1]^{K}$.

For $K \geq 2$, there are several types of queries we might be interested in (actually many of these queries make sense for $K=1$ too). Among them, we have:

- Exact match queries: given a key $\mathbf{x}$, determine whether a record with key $\mathbf{x}$ is present in the file $F$ or not.

- Range queries: given a hyperrectangle $\left[\ell_{0}, u_{0}\right] \times\left[\ell_{1}, u_{1}\right] \times \cdots \times\left[\ell_{K-1}, u_{K-1}\right]$, return the subset of records in $F$ which fall inside the given hyperrectangle, i.e., those keys $\mathbf{x}$ such that $\ell_{j} \leq x^{(j)} \leq u_{j}$ for all $0 \leq j<K$.

- Nearest neighbor queries: given a key $\mathbf{x}$, find the $m$ keys in the file closest to the given one, according to some "distance" measure (we should better say a similarity measure) defined over $D$.

- Partial match queries: given a query $q=\left(q_{0}, q_{1}, \ldots, q_{K-1}\right)$ where each $q_{j}$ is either a value in $D_{j}$ (specified) or $q_{j}=*$ (unspecified), return the subset of records in $F$ whose attributes coincide with the specified attributes of $q$, i.e., return $\mathbf{x}$ if and only if, for any $j, q_{j}=*$ or $q_{j}=x^{(j)}$.

In this paper we analyze the performance of partial match queries for several different multidimensional data structures. Before going on, and describing these data structures, we fix a piece of notation that will be consistently used through the paper. The number of specified attributes in a partial match query will be denoted $s$. We assume that $0<s<K$. Otherwise, when $s=K$, we have exact match queries; and if $s=0$, all keys in the file satisfy the query. As we will see, the performance of partial match depends on the ratio $\rho=s / K$ rather than on the actual values of $s$ and $K$. By hypothesis, $0<\rho<1$.

In the last 20 years, several data structures have been proposed for the dynamic maintenance of files of multidimensional records (i.e., the file must support both insertions and deletions) and a variety of associative queries. Clearly, it is desirable that any multidimensional data structure efficiently supports several associative queries, rather than being tailor-made for just one type of associative query.

$K$-dimensional binary search trees ( $K$ d-trees, for short) were introduced by Bentley [1] as a practical solution to this problem. Algorithms for $K$ d-trees are simple, robust, flexible, and usually exhibit good average performance. All the associative queries mentioned above are efficiently supported by $K$ d-trees.

A $K$ d-tree for a file $F$ can be incrementally built by successive insertions into an initially empty $K$ d-tree as follows. The first key is put into a single node with two empty subtrees. The first attribute of the second key is compared then with the first attribute of the key at the root: if it is smaller or equal, the second key is recursively inserted in the (empty) left subtree; otherwise, it is recursively inserted in the (empty) right subtree. The first attribute of the third key is compared with the first attribute of the key at the root, and recursively inserted into the left or right subtree, as before. However, if the subtree is not empty (because it contains the second key), we shall not compare the respective first attributes of the keys, but their second attributes. In general, when inserting a key $\mathbf{x}$, we compare the key to be inserted with the key $\mathbf{z}$ at the root of some subtree: if $\mathbf{z}$ is 


$$
\begin{aligned}
& x_{1}=(0.692,0.703) \\
& x_{2}=(0.286,0.495) \\
& x_{3}=(0.410,0.895) \\
& x_{4}=(0.522,0.953) \\
& x_{5}=(0.507,0.394) \\
& x_{6}=(0.295,0.300) \\
& x_{7}=(0.811,0.605) \\
& x_{8}=(0.912,0.807) \\
& x_{9}=(0.093,0.210) \\
& x_{10}=(0.188,0.109)
\end{aligned}
$$
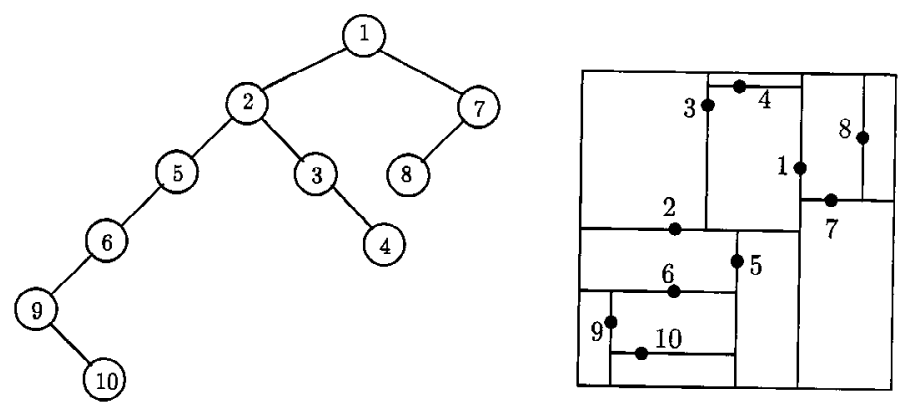

Fig. 1. A 2d-tree and its corresponding partition of the search space.

at level $j$, we compare $x^{(j \bmod K)}$ and $z^{(j \bmod K)}$, and recursively continue the insertion in the left or the right subtree of $\mathbf{z}$, until a leaf (empty subtree) is found, assuming that $\mathbf{x}$ is not already present in the tree. In Figure 1 we depict the $2 \mathrm{~d}$-tree that results from the insertion of $N=10$ keys into an initially empty tree, in the same order as they have been listed in the left part of the figure. The figure also shows the partition of $[0,1]^{2}$ induced by the $2 \mathrm{~d}$-tree. It should be clear that if the same points were inserted in a different order they could yield a substantially different $2 \mathrm{~d}$-tree.

Exact match queries in $K$ d-trees obviously operate by following a path down the tree, exactly the same way as if we were inserting that key: either we find it and report success or we reach a leaf, reporting failure.

The standard model for the probabilistic analysis of $K \mathrm{~d}$-trees is that a random $K \mathrm{~d}$ tree of size $N$ is built by inserting $N$ points independently drawn from some continuous probability distribution defined over $[0,1]^{K}$. This is equivalent to assuming that the probability that an insertion falls in a given leaf of a random $K$ d-tree of size $N$ is the same for any of its $N+1$ leaves. Under this assumption, the performance of updates and exact match queries coincides with the performance of updates and searches in binary search trees (notice that 1d-trees and binary search trees are the same); therefore, the expected cost of updates and exact match queries in random $K$ d-trees is $\mathcal{O}(\log n)$. Furthermore, these costs are independent of $K$. For partial match queries, Flajolet and Puech [7] have shown that the average cost of a partial match query $q$ with $s$ out of $K$ specified attributes is

$$
\mathbb{E}\left[C_{n}\right]=\beta_{q} n^{1-\rho+\vartheta(\rho)}+o\left(n^{1-\rho+\vartheta(\rho)}\right),
$$

where $\vartheta(x)$ is the unique real solution of

$$
(\vartheta(x)+3-x)^{x}(\vartheta(x)+2-x)^{1-x}-2=0,
$$

and $\beta_{q}$ is a constant which only depends on the pattern of alternance between specified and unspecified attributes in $q$. Furthermore, for $0<x<1, \vartheta(x) \leq 0.07$, but the result above disproved an old conjecture that $\mathbb{E}\left[C_{n}\right]=\Theta\left(n^{1-\rho}\right)$.

The idea of $K$ d-trees can easily be generalized to digital search trees: instead of partitioning the search space using the data, we can make a regular partition of the search space, based on the digits. The recursive partition of a region of the search space 


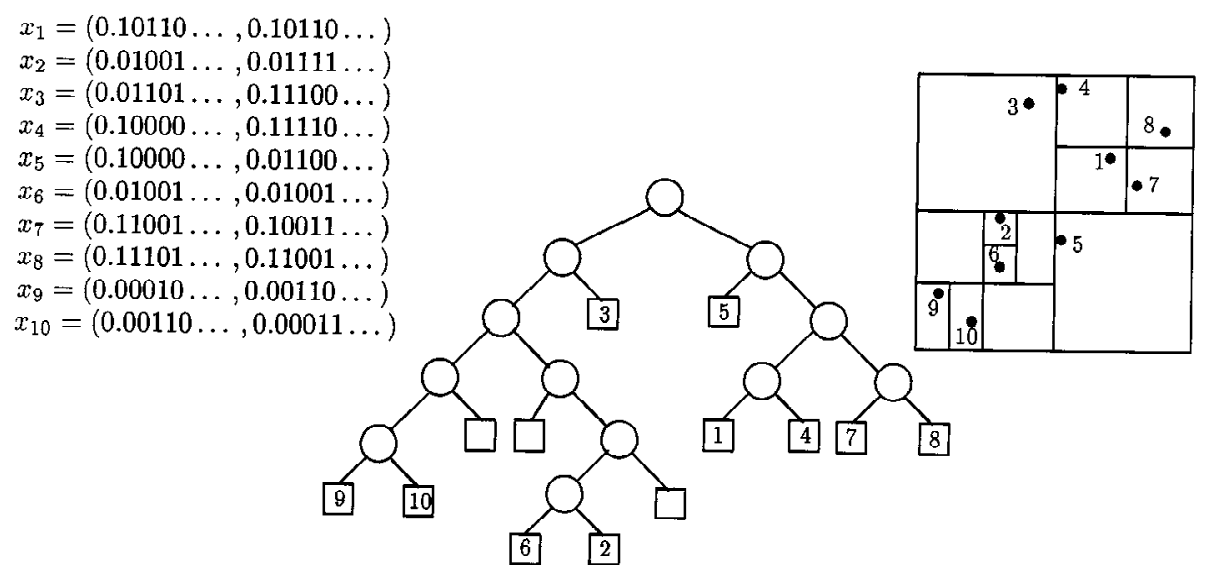

Fig. 2. A 2d-trie and its corresponding partition of the search space.

is not continued further when it contains one or no data points. Thus in a search in a binary $K$ d-trie we use the first bit of the first attribute of the given key in the first level, going to the left if it were a zero, and to the right if it were a one. Then we use the first bit of the second key in the second level, the first bit of the third key, and so on, down to the $(K-1)$ th level. Then, in the $K$ th level, we use the second bit of the first key, etc. In general, in the $j$ th level we must use the $\lfloor j / K\rfloor$ th bit of the $(j \bmod K)$ th attribute of the given key. Figure 2 depicts a 2d-trie for the same set of keys (now expressed in binary) as in Figure 1, and the induced partition of the search space. Notice that both the tree and the partition depend on the given set of keys but not on the order those were inserted.

Digital multidimensional data structures were first proposed around 1975 [2], [15] and further analyzed in [7], [11], [12], and [18]. The performance of partial match in $K$ d-tries and other multidimensional digital data structures (digital search trees, Patricia) is on the average $\Theta\left(n^{1-\rho}\right)$, the only difference being the coefficient-which is not constant, but a fluctuating function - of the main order term. The usual probability model for the analysis of these digital data structures, the so-called Bernoulli model, is that each of the $K$ attributes of a key $\mathbf{x}$ is an independently generated infinite string of 0 's and 1's, with each bit independently generated from the others, with identical probability $1 / 2$ (symmetric Bernoulli model) or with fixed probabilities $p$ and $q=1-p$ (asymmetric Bernoulli model).

Quite recently, Duch et al. [4] have used a technique called relaxation to obtain a variant of $K$ d-trees that facilitates the introduction of randomization, rotations, and other similar operations which tend to be complex and inefficient in the standard variant. The relaxed multidimensional trees differ from their standard counterparts because in the relaxed trees each node explicitly stores a value $j$ that will be used as the discriminator when a search path "passes" through that node. So, instead of using the preceptive attribute given by the cyclic rule of standard $K$ d-trees, in relaxed $K$ d-trees a field of the node is examined to know what attribute has to be used for the comparison at that point. This idea was already used by Friedman et al. [9] in their optimized $\mathrm{Kd}$-trees, where explicitly stored discriminators are used to build an optimal $K$ d-tree, a fortiori, 
given all the keys in the file in advance, and under the assumption that neither insertions nor deletions are made afterwards. In [4], however, relaxed $K$ d-trees are used for the dynamic maintenance of a file, and the discriminator assigned to each node is given by an independent random variable uniformly distributed over $\{0, \ldots, K-1\}$.

Random relaxed $K$ d-trees thus satisfy the following recursive definition: $T$ is a random relaxed $K d$-tree if and only if

1. $|T|=0$ (i.e., $T$ is empty) or

2. $|T|=n$, its left subtree $L$ is a random relaxed $K$ d-tree of size $i, 0 \leq i<n$, its right subtree $R$ is a random relaxed $K$ d-tree of size $n-1-i$, and for any $\mathbf{x} \in T$ and any discriminator $j, 0 \leq j<K$,

$$
\mathbb{P}[\langle\mathbf{x}, j\rangle \text { is the root of } T]=\frac{1}{n K} .
$$

Random $K$ d-trees satisfy a similar (at least in spirit) but more complex recursive characterization, as the discriminators in successive levels must follow the cyclic sequence: $0,1, \ldots, K-1,0,1, \ldots$

As we shall see the recursive definition above makes the analysis of partial match in relaxed $K$ d-trees much easier. We tackle this analysis in Sections $2-4$.

We prove that the expected cost of a partial match in a random relaxed $K$ d-tree is $\beta \cdot n^{\varepsilon}+\mathcal{O}(1)$ (Theorems 1 and 2), where $\varepsilon$ and $\beta$ are constants only depending on $\rho$. Relaxed $K$ d-trees perform slightly worse than $K$ d-trees, as $\varepsilon(\rho) \geq 1-\rho+\vartheta(\rho)$ for all $\rho$, but the difference is almost insignificant (the difference between $\varepsilon$ and $1-\rho+\vartheta(\rho)$ is always "small," never exceeding 0.08). Also, the constant $\beta$ for relaxed $K$ d-trees is independent of the query pattern, while for standard $K$ d-trees there is a possibly distinct constant $\beta_{q}$ for each of the $2^{K}-2$ relevant query patterns $q$. Thus relaxed $K \mathrm{~d}$-trees are not sensitive to the actual query, whereas $K$ d-trees are, and the difference in the performance between two different partial match queries on $K$ d-trees could be (for values of $n$ in practical ranges) of the same order of magnitude or even greater than the differences of performance between $K$ d-trees and relaxed $K$ d-trees.

Next, in Section 4, we compute the variance of the cost of partial match in $K \mathrm{~d}$ trees, which is $\beta^{\prime} \cdot n^{2 \varepsilon}+\mathcal{O}\left(n^{\varepsilon}\right)$. In Section 5 we make a slight detour from the main theme of the paper, and briefly consider the analysis of the variance of the cost of partial match in quadtrees, a rather different multidimensional data structure, whose analysis has remarkable similarities with that of $K$ d-trees (both standard and relaxed). We find the variance for two-dimensional quadtrees. The variance for higher dimensional quadtrees still remains open.

The following sections (Sections 6-9) cover the analysis of partial match in relaxed multidimensional digital trees, which can be defined analogously to relaxed $K$ d-trees. In particular, we analyze the expected cost of partial match in random relaxed $K \mathrm{~d}$-tries and its variance, and compare the expected costs for the relaxed variants of $K \mathrm{~d}$-tries, $K$ d-digital search trees, and $K$ d-Patricia.

2. Partial Match in Relaxed Kd-Trees. Let $C_{n}$ be the cost of partial match in a random relaxed $K$ d-tree of size $n$ when $s$ out of the $K$ attributes have been specified, with $0<s<K$, and let $\rho=s / K$. 
The analysis of partial match in standard $K$ d-trees explicitly involves the query pattern and requires the solution of a system of $K$ equations [7]. On the contrary, the analysis of partial match in relaxed $K$ d-trees is considerably less difficult than the analysis of partial match in standard $K$ d-trees, because it is equivalent to the analysis of a "valuation" $\operatorname{pm}(\cdot)$ over random binary search trees. Let $\operatorname{pm}(t)$ denote the cost of a partial match query in a relaxed $K$ d-tree with shape $t$. This random variable is defined by

$$
\begin{aligned}
\mathbb{P}[\operatorname{pm}(t)=k]= & \rho\left(\frac{\left|t_{1}\right|+1}{|t|+1} \mathbb{P}\left[\operatorname{pm}\left(t_{1}\right)=k-1\right]+\frac{\left|t_{2}\right|+1}{|t|+1} \mathbb{P}\left[\operatorname{pm}\left(t_{2}\right)=k-1\right]\right) \\
& +(1-\rho) \mathbb{P}\left[\operatorname{pm}\left(t_{1}\right)+\operatorname{pm}\left(t_{2}\right)=k-1\right],
\end{aligned}
$$

if $t=\circ\left(t_{1}, t_{2}\right)$ and $k>0, \mathbb{P}[\operatorname{pm}(\square)=0]=1$ and $\mathbb{P}[\operatorname{pm}(t)=k]=0$ in any other case.

The recurrence above is simply the formalization of the following intuitive argument: if the given tree is not empty, then with probability $(1-\rho)$ the root of the tree is not specified and one needs to visit the root and then continue the search in both subtrees; on the other hand, with probability $\rho$ the root of the tree is specified and one needs to visit the root and then proceed recursively on the left subtree with probability $(\ell+1) /(n+1)$ (where $\ell$ is the size of the left subtree and $n$ the size of the tree) and on the right subtree with complementary probability.

PROPOSITION 1. Let $C(z, u)$ be the bivariate generating function

$$
C(z, u)=\sum_{n \geq 0} \sum_{k \geq 0} \mathbb{P}\left[C_{n}=k\right] z^{n} u^{k} .
$$

It satisfies

$$
\frac{\partial^{2}}{\partial z^{2}} z C(z, u)=\frac{2 \rho}{1-z} u \frac{\partial}{\partial z} z C(z, u)+\frac{1-\rho}{z} u \frac{\partial}{\partial z}(z C(z, u))^{2}
$$

with the initial conditions $C(0, u)=1$ and $C_{z}(0, u)=u$. Here and in what follows $C_{x}(z, u)$ denotes the partial derivative of $C(z, u)$ with respect to the variable $x$.

PROOF. To find the functional equation satisfied by $C(z, u)$, we first introduce the convenient and appealing notation

$$
u^{\mathrm{pm}(t)}=\sum_{k \geq 0} \mathbb{P}[\mathrm{pm}(t)=k] u^{k},
$$

for the probability generating function associated to the random variable $\mathrm{pm}(t)$.

Then the recurrence for $\mathbb{P}[\operatorname{pm}(t)=k]$ translates to

$$
u^{\mathrm{pm}(t)}=u \cdot\left(\rho \frac{\left|t_{1}\right|+1}{|t|+1} u^{\mathrm{pm}\left(t_{1}\right)}+\rho \frac{\left|t_{2}\right|+1}{|t|+1} u^{\mathrm{pm}\left(t_{2}\right)}+(1-\rho) u^{\mathrm{pm}\left(t_{1}\right)+\mathrm{pm}\left(t_{2}\right)}\right),
$$

if $t$ is nonempty, and $u^{\mathrm{pm}(\square)}=1$. 
We may now symbolically write, for $C(z, u)$,

$$
C(z, u)=\sum_{t} \lambda(t) z^{|t|} u^{\mathrm{pm}(t)}
$$

where the sum extends to all binary trees $t$, and $\lambda(t)$ is the probability that a random permutation produces the binary search tree $t$ [14], [20],

$$
\lambda(t)= \begin{cases}1 & \text { if } t \text { is empty, } \\ \frac{\lambda\left(t_{1}\right) \cdot \lambda\left(t_{2}\right)}{|t|} & \text { if } t=\circ\left(t_{1}, t_{2}\right) .\end{cases}
$$

Using the recursions for $\lambda(t)$ and $u^{\mathrm{pm}(t)}$ we get, by routine algebraic manipulations, the statement of the proposition.

Proposition 1 is the basis for our study, as successive differentiation of $C(z, u)$ with respect to $u$ and setting $u=1$ will yield the corresponding differential equations satisfied by the different generating functions for factorial moments, including the generating function of expectations (the factorial moment of order 1).

3. The Average Cost. To compute the expectation of $C_{n}$ we first introduce the generating function

$$
C_{1}(z)=\sum_{n \geq 0} \mathbb{E}\left[C_{n}\right] z^{n}
$$

PROPOSITION 2. The generating function $C_{1}(z)$ of the first moments of $C_{n}$ satisfies the following second-order linear differential equation:

$$
z C_{1}^{\prime \prime}(z)-2 \frac{(2 z-1)}{1-z} C_{1}^{\prime}(z)-2 \frac{2-\rho-z}{(1-z)^{2}} C_{1}(z)-2 \frac{1}{(1-z)^{3}}=0
$$

with the initial conditions $C_{1}(0)=0$ and $C_{1}^{\prime}(0)=1$.

PROOF. The statement of the proposition easily follows by differentiating with respect to $u$ and evaluating at $u=1$ both sides of Proposition 1 , as $C_{1}(z)=C_{u}(z, 1)$. The initial conditions can be found from simple reasoning about the combinatorics of the problem: $C_{0}=0$ and $C_{1}=1$.

PROPOSITION 3. The generating function $C_{1}(z)$ is

$$
C_{1}(z)=\frac{1}{1-\rho}\left(\frac{{ }_{2} F_{1}\left(\begin{array}{c}
a, b \\
2
\end{array} \mid z\right)}{(1-z)^{\alpha}}-\frac{1}{1-z}\right),
$$

where

$$
{ }_{2} F_{1}\left(\begin{array}{c}
a, b \\
c
\end{array} \mid z\right)={ }_{2} F_{1}(a, b ; c ; z)=\sum_{k \geq 0} \frac{a^{\bar{k}} b^{\bar{k}}}{c^{\bar{k}}} \frac{z^{k}}{k !}
$$


denotes the classical hypergeometric function [10], [21], and in this particular instance, $a=2-\alpha, b=1-\alpha$, and $\alpha=\frac{1}{2}+\frac{1}{2} \sqrt{9-8 \rho}$. Here $x^{\bar{k}}=x(x+1) \cdots(x+k-1)$, following Knuth's convention for rising factorials [13] (see also [10]).

PROOF. Using MAPLE ${ }^{5}$ it takes a matter of seconds to produce the following general solution to the differential equation of Proposition 2:

$$
\begin{aligned}
C_{1}(z)= & -\frac{1}{(1-\rho)(1-z)}+B_{1}(1-z)^{-\alpha}{ }_{2} F_{1}\left(\begin{array}{c}
a, b \\
2
\end{array} \mid z\right) \\
& +B_{2}(1-z)^{-\alpha}{ }_{2} F_{1}\left(\begin{array}{c}
a, b \\
2
\end{array} \mid z\right) \int_{z_{0}}^{z} \frac{(1-t)^{2 \alpha-2}}{t^{2}{ }_{2} F_{1}\left(\begin{array}{c}
a, b \\
2
\end{array} \mid t\right)} d t,
\end{aligned}
$$

where $\alpha=\frac{1}{2}+\frac{1}{2} \sqrt{9-8 \rho}, a=2-\alpha, b=1-\alpha$, and $B_{1}$ and $B_{2}$ are constants depending on the initial conditions, and the integration path from the starting point $z_{0}$ to $z$ must not encounter the logarithmic singularity at $z=0$.

Three points are worth noting here. First, if the initial conditions are supplied to MAPLE's dsolve, then it is not able to produce a solution. Second, different versions of MAPLE may give the solutions in rather different forms (or may not solve the equation at all). For instance, MAPLE V under Windows '95 gives the solution in terms of Legendre functions of the first kind. Third, the solution provided by MAPLE is expressed using powers of $(-1+z)$ which is clearly inconvenient for our needs. In this case we can change these to powers of $(1-z)$ by absorbing the appropriate factor into the arbitrary constants $B_{1}$ and $B_{2}$ of the general solution.

Once MAPLE has done the tough work, we only need to find the values of the constants $B_{1}$ and $B_{2}$. The last term has a logarithmic singularity at $z=0$, and hence the combinatorial nature of the problem excludes its contribution to $C_{1}(z)$, i.e., the constant $B_{2}$ must be 0 in this case. Now, using $C_{1}(0)=0$ we find that $B_{1}=1 /(1-\rho)$ and the statement of the proposition follows.

Although MAPLE saves us a lot of work here, we briefly explain how the solution could be found by hand. A similar development appears in the analysis of quadtrees by Flajolet et al. [5].

To begin with, it is not difficult to guess and check that $(1-\rho)^{-1}(1-z)^{-1}$ is a particular solution of the differential equation given in Proposition 2. From this starting point, we proceed by considering the general solution for the homogeneous equation. A natural step is to consider the substitution $g(z)=y(z) /(1-z)^{\alpha}$. This leads us to the conclusion that $\alpha$ has to be the solution of $-\alpha+\alpha^{2}-2(1-\rho)=0$, and that the differential equation for $y(z)$ is hypergeometric. Writing $y(z)=B_{1} y_{1}(z)+B_{2} y_{2}(z)$ for the general solution, the integral representation of $y_{2}(z)$ follows by applying the variation of constants method [21].

Undoing the substitution and adjusting the initial conditions, we finally arrive at the same closed form for $C_{1}(z)$ given in the proposition.

\footnotetext{
5 All references to the symbolic computation system MAPLE refer to version V, release 5, running under Solaris
} 5.5.1, unless otherwise indicated. 
THEOREM 1. Let $C_{n}$ denote the cost of a partial match query with s specified attributes out of $K$ in a random relaxed $K d$-tree of size $n$. Then

$$
\mathbb{E}\left[C_{n}\right]=-\frac{1}{1-\rho}+\frac{1}{1-\rho} \sum_{k=0}^{n}\left(\begin{array}{c}
n-k+\alpha-1 \\
n-k
\end{array}\right)\left(\begin{array}{c}
\alpha-1 \\
k
\end{array}\right)\left(\begin{array}{c}
\alpha-2 \\
k
\end{array}\right),
$$

where $\alpha=\frac{1}{2}+\frac{1}{2} \sqrt{9-8 \rho}$.

PROOF. The formula for the coefficients $\mathbb{E}\left[C_{n}\right]$ is just the convolution that follows from Proposition 3 and the binomial and hypergeometric expansions.

THEOREM 2. Let $C_{n}$ denote the cost of a partial match query with s specified attributes out of $K$ in a random relaxed $K d$-tree of size $n$. Then

$$
\mathbb{E}\left[C_{n}\right]=\beta n^{\alpha-1}+\mathcal{O}(1)
$$

where $\alpha$ is as in Theorem $1, a=1-\alpha, b=2-\alpha$, and

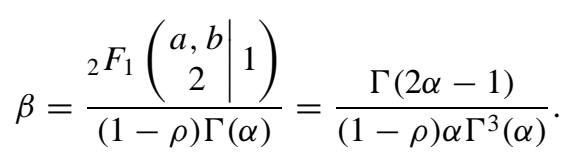

PROOF. To get the asymptotic behavior of $\mathbb{E}\left[C_{n}\right]$ it suffices to study the asymptotic behavior of $C_{1}(z)$ when $z \rightarrow 1$. The second term makes a contribution which can be neglected, and the hypergeometric function is analytic at $z=1$. Thus,

$$
C_{1}(z) \sim \beta^{\prime}(1-z)^{-\alpha}+\mathcal{O}\left((1-z)^{-1}\right),
$$

with $\beta^{\prime}=(1-\rho)^{-1} \cdot{ }_{2} F_{1}(a, b ; 2 ; 1)$ and from standard singularity analysis [6]

$$
\left[z^{n}\right] C_{1}(z)=\frac{\beta^{\prime}}{\Gamma(\alpha)} n^{\alpha-1}+\mathcal{O}(1)
$$

The hypergeometric function is analytic at $z=1$ and its value at this point can be found [10] because $\Re(c)=2>\Re(a)+\Re(b)=3-2 \alpha$. Indeed, for any $\rho, 0<\rho<1$, we have $1<\alpha<2$, and then $2>3-2 \alpha$. To evaluate ${ }_{2} F_{1}(a, b ; 2 ; 1)$ we apply the well known identity

$$
{ }_{2} F_{1}\left(\begin{array}{c|c}
a, b & 1 \\
c & 1
\end{array}\right)=\frac{\Gamma(c-a-b) \Gamma(c)}{\Gamma(c-a) \Gamma(c-b)} .
$$

It is interesting to point out that although Theorem 2 is valid only if $0<\rho<1$, it provides meaningful information for the limiting cases to some extent. If $\rho=0$, that is, no attribute is specified, then $\mathbb{E}\left[C_{n}\right]=n$. Indeed, $\alpha-1 \rightarrow 1$ and $\beta \rightarrow 1$ as $\rho \rightarrow 0$. On the other hand, in an exact match all attributes are specified and $\rho=1$. In this case, we know that $\mathbb{E}\left[C_{n}\right]=\mathcal{O}(\log n)$, and we have that $\alpha-1 \rightarrow 0$ and $\beta \rightarrow \infty$ if $\rho \rightarrow 1$, which is an approximate way to express by a formula of the type $\beta \cdot n^{\alpha-1}$ that $\mathbb{E}\left[C_{n}\right]$ grows with $n$, but slower than any function of the type $n^{\varepsilon}$, for positive $\varepsilon$. 


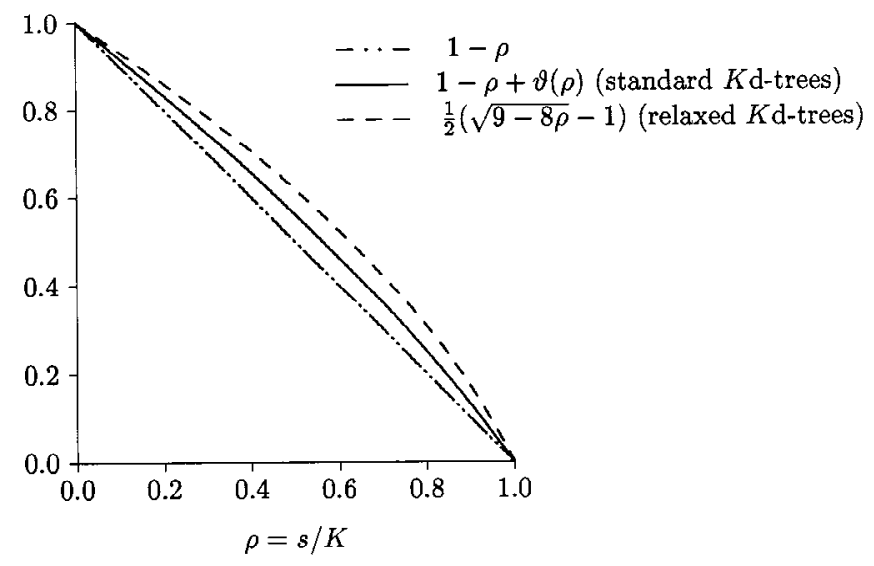

Fig. 3. The value of the exponent in the average cost of partial match in standard and relaxed $K d$-trees.

In Figure 3 we plot $1-\rho+\vartheta(\rho)$ against $\alpha(\rho)-1$, the exponents in the main order term of the average cost of partial match in standard and relaxed $K$ d-trees, respectively. The plot shows that standard $K$ d-trees perform slightly better than relaxed $K$ d-trees for all values of $\rho$.

An intuitive explanation for this difference is that in standard $K$ d-trees a partial match will follow, for every $K$ steps, one branch $s$ times and both branches $K-s$ times, whereas these figures hold only on the average in relaxed $K d$-trees. Since there is a nonnull probability that the event "both subtrees are explored" occurs more than $K-s$ times in $K$ steps, the expected cost of a partial match in relaxed $K$ d-trees is larger. Relaxed $K$ d-trees exhibit other advantages, though: their average performance does not depend on the actual pattern of the query, and they are less sensitive to deviations from the hypothesis of "anisotropy," i.e., no coordinate axis is privileged over the others.

4. The Variance. The steps we follow to find $\mathbb{V}\left[C_{n}\right]$ are basically the same as in the previous section. Alas, it will be considerably more difficult to find a closed form for $C_{2}(z)$, the generating function for the second factorial moments of $C_{n}$ :

$$
C_{2}(z)=\sum_{n \geq 0} \mathbb{E}\left[C_{n}^{\frac{2}{n}}\right] z^{n} .
$$

In the formula above, we use Knuth's convention for falling factorials: $x^{2}=x(x-1)$, and in general, $x \underline{k}=x \cdot(x-1) \cdots(x-k+1)$.

For the rest of this section, we define $\alpha=(1+\Delta) / 2, \Delta=\sqrt{9-8 \rho}, a=2-\alpha$, and $b=1-\alpha$, thus bearing the same meanings as in the previous section.

PROPOSITION 4. The generating function $C_{2}(z)$ of the second factorial moments of $C_{n}$ satisfies the second-order linear differential equation

$$
C_{2}^{\prime \prime}(z)-\frac{2(2 z-1)}{z(1-z)} C_{2}^{\prime}(z)-\frac{2(2-\rho-z)}{z(1-z)^{2}} C_{2}(z)=s(z)
$$


where the inhomogeneous part is

$$
\begin{aligned}
s(z)= & \frac{2(2-z+z \Delta) \cdot{ }_{2} F_{1}\left(\begin{array}{c}
a, b \\
2
\end{array} \mid z\right)}{(1-\rho) z(1-z)^{2 \alpha+1}}-\frac{4 \rho \cdot{ }_{2} F_{1}\left(\begin{array}{c}
a, b \\
2
\end{array} \mid z\right)}{(1-\rho) z(1-z)^{\alpha+2}} \\
& -\frac{2(\Delta+2 \rho-3) \cdot{ }_{2} F_{1}\left(\begin{array}{c}
a, b \\
2
\end{array} \mid z\right) \cdot{ }_{2} F_{1}\left(\begin{array}{c}
a+1, b+1 \\
3
\end{array} \mid z\right)}{(1-\rho)(1-z)^{2 \alpha}}-\frac{4}{z(1-z)^{3}}
\end{aligned}
$$

and the initial conditions are $C_{2}(0)=0$ and $C_{2}^{\prime}(0)=0$.

Proof. Differentiating the equation given in Proposition 1 twice with respect to $u$ and evaluating at $u=1$ leads to the given equation for $C_{2}(z)=C_{u u}(z, 1)$. Also, we use the result of Proposition 3 in the derivation of this equation.

PROPOSITION 5. The generating function $\mathrm{C}_{2}(z)$ is

$$
C_{2}(z)=\frac{1}{(1-z)^{\alpha}} \cdot{ }_{2} F_{1}\left(\begin{array}{c}
a, b \\
2
\end{array} \mid z\right) \int_{0}^{z}\left(\int_{0}^{x} \frac{t_{2}^{2} F_{1}\left(\begin{array}{c}
a, b \\
2
\end{array} \mid t\right) s(t) d t}{(1-t)^{\alpha-2}}\right) \frac{(1-x)^{2 \alpha-2} d x}{x_{2}^{2}{ }_{2} F_{1}^{2}\left(\begin{array}{c}
a, b \\
2
\end{array} \mid x\right)} .
$$

PROOF. Notice that the homogeneous equation corresponding to the differential equation in Proposition 5 is the same as the homogeneous differential equation corresponding to that given in Proposition 3, but we do not have a particular solution for the inhomogeneous differential equation nor we can disregard the logarithmic term in the general solution of the homogeneous equation.

In principle, we can find a solution to the differential equation satisfied by $C_{2}(z)$ using MAPLE. However, we prefer to illustrate here how to get the solution by hand. An additional reason to discard MAPLE's solution is that we are looking for an integral representation of $C_{2}(z)$ which will be more useful than that provided by MAPLE: it computes an integral representation for $C_{2}(z)$ if the independent term $s(z)$ is not specified, i.e., we make a call to dsolve(homogeneous equation $=\mathrm{s}(\mathrm{z}), \mathrm{C}(\mathrm{z})$ ), where $s(z)$ is some unspecified function. However, the form of the solution given by MAPLE is less convenient for later manipulations.

So we start from the general solution of the homogeneous differential equation

$$
g(z)=\frac{B_{1}}{(1-z)^{\alpha}} 2 F_{1}\left(\begin{array}{c}
a, b \\
2
\end{array} \mid z\right)+\frac{B_{2}}{(1-z)^{\alpha}} 2 F_{1}\left(\begin{array}{c}
a, b \\
2
\end{array} \mid z\right) \int_{z_{0}}^{z} \frac{(1-t)^{2 \alpha-2} d t}{t_{2}^{2} F_{1}^{2}\left(\begin{array}{c}
a, b \\
2
\end{array} \mid t\right)}
$$

and apply the variation of constants method to get a closed form for $C_{2}(z)$.

That means we write one particular solution for the equation given in the proposition as

$$
g_{p}(z)=B_{p, 1}(z) g_{1}(z)+B_{p, 2}(z) g_{2}(z) .
$$

This leads to the following system of linear equations for the derivatives $B_{p, 1}^{\prime}(z)$ and $B_{p, 2}^{\prime}(z)$ :

$$
B_{p, 1}^{\prime}(z) g_{1}(z)+B_{p, 2}^{\prime}(z) g_{2}(z)=0 \quad \text { and } \quad B_{p, 1}^{\prime}(z) g_{1}^{\prime}(z)+B_{p, 2}^{\prime}(z) g_{2}^{\prime}(z)=s(z) .
$$


With the solutions

$$
B_{p, 2}^{\prime}(z)=\frac{s(z)}{g_{1}(z) \widehat{g}(z)} \quad \text { and } \quad B_{p, 1}^{\prime}(z)=-B_{p, 2}^{\prime}(z) \int_{z_{0}}^{z} \widehat{g}(t) d t
$$

we get one particular solution of the differential equation by integration and resubstitution, namely,

$$
\begin{aligned}
g_{p}(z) & =g_{1}(z) \int_{z_{0}}^{z}\left(\int_{x_{0}}^{x} \frac{s(t)}{g_{1}(t) \widehat{g}(t)} d t\right) \widehat{g}(x) d x \\
& =\frac{1}{(1-z)^{\alpha}}{ }_{2} F_{1}\left(\begin{array}{c}
a, b \\
2
\end{array} \mid z\right) \int_{z_{0}}^{z}\left(\int_{x_{0}}^{x} \frac{t^{2}{ }_{2} F_{1}\left(\begin{array}{c}
a, b \\
2
\end{array} \mid t\right) s(t) d t}{(1-t)^{\alpha-2}}\right) \frac{(1-x)^{2 \alpha-2} d x}{x^{2}{ }_{2} F_{1}^{2}\left(\begin{array}{c}
a, b \\
2
\end{array}\right)} .
\end{aligned}
$$

Adapting the initial conditions can be done now by choosing the starting points of the integration paths $x_{0}$ and $z_{0}$, thus getting the claimed integral representation of $C_{2}(z)$.

THEOREM 3. Let $C_{n}$ denote the cost of a partial match query with s specified attributes out of $K$ in a random relaxed $K d$-tree of size $n$. Then

$$
\mathbb{V}\left[C_{n}\right] \sim\left(\frac{8 \Gamma(2 \alpha)}{\alpha^{2}(\alpha-1)^{2}(2 \alpha-1)(3 \alpha-2) \Gamma^{4}(\alpha)}-\frac{4 \Gamma^{2}(2 \alpha)}{\alpha^{4}(\alpha-1)^{2}(2 \alpha-1)^{2} \Gamma^{6}(\alpha)}\right) n^{2 \alpha-2} .
$$

PROOF. The coefficients $\mathbb{E}\left[C_{n}^{\frac{2}{n}}\right]=\left[z^{n}\right] C_{2}(z)$ can now be found asymptotically by means of singularity analysis, from its integral representation (Proposition 5).

First we get a local expansion for $s(z)$ around $z=1$, namely,

$$
\begin{aligned}
& s(z)=\frac{4 \alpha_{2} F_{1}^{2}\left(\begin{array}{c|c}
a, b & 1 \\
2 & 1
\end{array}\right)}{(1-\rho)(1-z)^{2 \alpha+1}}+\mathcal{O}\left(\frac{1}{(1-z)^{\alpha+2}}\right) \\
& =\frac{4 \alpha \Gamma^{2}(2 \alpha-1)}{(1-\rho) \Gamma^{2}(\alpha) \Gamma^{2}(\alpha+1)(1-z)^{2 \alpha+1}}+\mathcal{O}\left(\frac{1}{(1-z)^{\alpha+2}}\right) .
\end{aligned}
$$

Notice that $\alpha+2<2 \alpha+1$, so the asymptotic expansion is valid. Furthermore, this last inequality implies that the initial conditions and the arising integration constants do not match, so we get

$$
\begin{aligned}
C_{2}(z)= & \frac{1}{(1-z)^{\alpha}} \int_{0}^{z}\left(\int_{0}^{x} \frac{B_{3} d t}{(1-t)^{3 \alpha-1}}\right)(1-x)^{2 \alpha-2} d x \\
& +\mathcal{O}\left(\max \left\{\frac{1}{(1-z)^{\alpha}}, \frac{1}{(1-z)^{2 \alpha-2}}\right\}\right) \\
= & \frac{B_{3}}{(3 \alpha-2)(\alpha-1)} \frac{1}{(1-z)^{2 \alpha-1}}+\mathcal{O}\left(\max \left\{\frac{1}{(1-z)^{\alpha}}, \frac{1}{(1-z)^{2 \alpha-2}}\right\}\right),
\end{aligned}
$$

where $B_{3}$ is the constant factor of the first term in the asymptotic expansion of $s(z)$. 


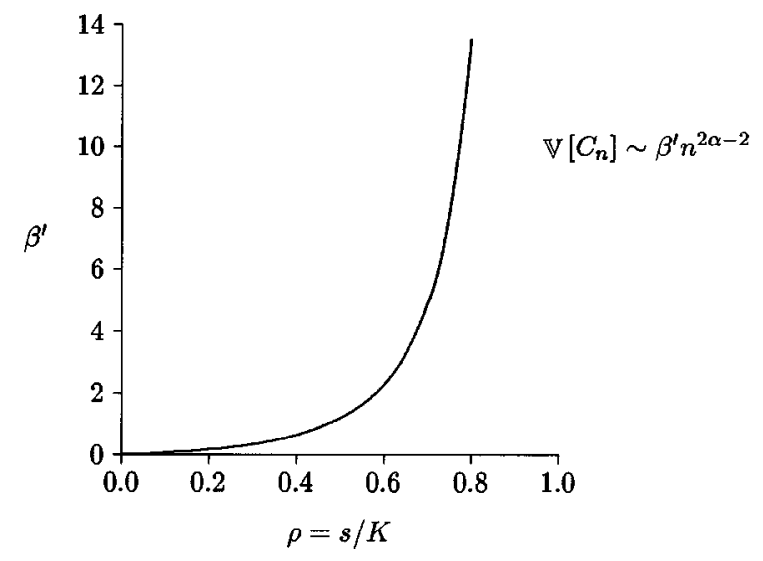

Fig. 4. The value of the constant $\beta^{\prime}$ in the variance of partial match in relaxed $K$ d-trees.

This leads to the following asymptotic estimate for the coefficients:

$$
\begin{aligned}
\mathbb{E}\left[C_{n}^{\frac{2}{n}}\right] & =\left[z^{n}\right] C_{2}(z)=\frac{B_{3}}{(3 \alpha-2)(\alpha-1)} \frac{n^{2 \alpha-2}}{\Gamma(2 \alpha-1)}+\mathcal{O}\left(\max \left\{n^{\alpha-1}, n^{2 \alpha-3}\right\}\right) \\
& =\frac{8 \Gamma(2 \alpha) n^{2 \alpha-2}}{\alpha^{2}(\alpha-1)^{2}(2 \alpha-1)(3 \alpha-2) \Gamma^{4}(\alpha)}+\mathcal{O}\left(n^{\alpha-1}\right) .
\end{aligned}
$$

The asymptotic equivalent for the variance given in the statement of the theorem is easily computed from the known asymptotic estimates for $\mathbb{E}\left[C_{n}\right]$ (Theorem 2), from the ones we just have obtained for $\mathbb{E}\left[C_{n}^{\frac{2}{n}}\right]$, and

$$
\mathbb{V}\left[C_{n}\right]=\mathbb{E}\left[C_{n}^{\frac{2}{n}}\right]+\mathbb{E}\left[C_{n}\right]-\left(\mathbb{E}\left[C_{n}\right]\right)^{2} .
$$

The order of magnitude of the variance for relaxed $K$ d-trees is the square of that of its corresponding expected value; this phenomenon also occurs in standard $K$ d-trees, where the variance is $\Theta\left(n^{2(1-\rho+\vartheta(\rho))}\right)$ [3].

5. A Note on Two-Dimensional Quadtrees. We cannot refrain from making a few comments about quadtrees, as the analysis of partial match in quadtrees so closely resembles the analysis performed in the two previous subsections. In [5] the expected cost of partial match in quadtrees of arbitrary dimension was explicitly computed by means of the techniques we have sketched in previous sections. However, the variance of partial match in quadtrees, even for the particular case $K=2$, was unknown. We consider here this particular case and compute this variance $\mathbb{V}\left[Q_{n}\right]$ using the arguments of the previous subsection almost verbatim. Notice that a partial match in dimension $K=2$ means that exactly one coordinate is specified while the other is left unspecified, that is, $\rho=\frac{1}{2}$. 
THEOREM 4. Let $Q_{n}$ be the cost of a partial match in a random 2-quadtree of size $n$. Then

$$
\mathbb{V}\left[Q_{n}\right] \sim \beta n^{\varepsilon}=\left(\frac{(2 \alpha-1) \Gamma(2 \alpha)}{3 \alpha(\alpha-1) \Gamma^{4}(\alpha)}-\frac{\Gamma^{2}(2 \alpha)}{4 \Gamma^{6}(\alpha)}\right) n^{2 \alpha-2}
$$

where

$$
\begin{aligned}
\alpha & =\frac{\sqrt{17}-1}{2} \approx 1.561552813 \ldots \\
\beta & \approx 0.3493015 \ldots, \quad \text { and } \\
\varepsilon & =2 \alpha-2 \approx 1.12310526 \ldots
\end{aligned}
$$

PROOF. We do not show a full proof of the theorem here, but rather sketch it. Let $Q_{2}(z)$ be the generating function for the second factorial moment of $Q_{n}$. The recurrence for these costs translates to

$$
Q_{2}^{\prime \prime}(z)+\frac{2}{z} Q_{2}^{\prime}(z)-\frac{4}{z(1-z)^{2}} Q_{2}(z)=s(z),
$$

where the initial conditions are $Q_{2}(0)=0$ and $Q_{2}^{\prime}(0)=0$. From there, and in a manner similar to that shown in the proof of Theorem 3 , we find

$$
\begin{aligned}
Q_{2}(z)= & \frac{1}{(1-z)^{\alpha}}{ }_{2} F_{1}\left(\begin{array}{c}
1-\alpha,-\alpha \\
2
\end{array} \mid z\right) \int_{0}^{z}\left(\int_{0}^{x} \frac{t^{2}{ }_{2} F_{1}\left(\begin{array}{c}
1-\alpha,-\alpha \\
2
\end{array} \mid t\right) s(t) d t}{(1-t)^{\alpha}}\right) \\
& \times \frac{(1-x)^{2 \alpha} d x}{x_{2}^{2} F_{1}^{2}\left(\begin{array}{c}
1-\alpha,-\alpha \\
2
\end{array} \mid x\right)}
\end{aligned}
$$

where now $\alpha$ is the solution of the indicial equation, given by $\alpha=(\sqrt{17}-1) / 2$ and

$$
s(z)=\frac{4}{z(1-z)^{2 \alpha+1}} 2 F_{1}^{2}\left(\begin{array}{c}
1-\alpha,-\alpha \\
2
\end{array} \mid z\right)-\frac{4}{z(1-z)^{3}} .
$$

The third and final step is to work out asymptotic expansions for $\mathbb{E}\left[Q_{n}^{\frac{2}{n}}\right]$ as in Section 4 and then for $\mathbb{V}\left[Q_{n}\right]$.

6. Partial Match in Relaxed $K \mathbf{K d}$-Tries. As we did for relaxed $K$ d-trees in the previous sections, it is convenient to start defining a bivariate generating function $C(z, u)$ associated to the costs $C_{n}$ of a partial match in a random relaxed $K$ d-trie of size $n$, where $s$ out of the $K$ attributes have been specified. Again, $\rho=s / K$ and $0<\rho<1$. However, the analysis of $K$ d-tries makes it advisable to define $C(z, u)$ as the exponential generating function of the probability generating functions $C_{n}(u)=n !\left[z^{n}\right] C(u, z)=$ $\sum_{k \geq 0} \mathbb{P}\left[C_{n}=k\right] u^{k}$ rather than the ordinary generating function of the $C_{n}(u)$ 's. 
PROPOSITION 6. Let $C(z, u)$ be the bivariate generating function

$$
C(z, u)=\sum_{n \geq 0} \sum_{k \geq 0} \mathbb{P}\left[C_{n}=k\right] u^{k} \frac{z^{n}}{n !} .
$$

It satisfies

$$
C(z, u)=\rho u e^{z / 2} C\left(\frac{z}{2}, u\right)+(1-\rho) u C^{2}\left(\frac{z}{2}, u\right)+(1-u)(1+z) .
$$

PROOF. It is more or less immediate from the recursion of $\mathrm{pm}(\cdot)$ and the Bernoulli model: the bits of the multidimensional keys are independently generated and with symmetric probability, i.e.,

$$
\mathbb{P}\left[\text { the } i \text { th bit of } x^{(j)}=0\right]=\mathbb{P}\left[\text { the } i \text { th bit of } x^{(j)}=1\right]=\frac{1}{2},
$$

for any $i$, any key $\mathbf{x}$, and any of its attributes $j, 0 \leq j<K$. Indeed, let

$$
C(z, u)=\sum_{t} \lambda(t) u^{\mathrm{pm}(t)} \frac{z^{|t|}}{|t| !},
$$

where the sum extends to all binary trees $t$ and $\lambda(t)$ now denotes the probability that $|t|$ random bitstrings produce a trie whose shape is $t$. For the Bernoulli model we have

$$
\lambda(t)= \begin{cases}1 & \text { if } \quad|t| \leq 1, \\
2^{-|t|}\left(\begin{array}{c}
|t| \\
\left|t_{1}\right|
\end{array}\right) \cdot \lambda\left(t_{1}\right) \cdot \lambda\left(t_{2}\right) & \text { if } \quad t=\circ\left(t_{1}, t_{2}\right) .\end{cases}
$$

The rest of the proof is routine algebraic manipulation.

The analysis of the expected value of $C_{n}$ and its variance follow from differentiation of $C(z, u)$ with respect to $u$ and setting $u=1$; but because of the difference-differential equation satisfied by $C(z, u)$, we would rather work with its Poisson transform $\hat{C}(z, u)$ of $C(z, u)$.

Proposition 7. The Poisson transform $\hat{C}(z, u)=e^{-z} C(z, u)$ satisfies

$$
\hat{C}(z, u)=\rho u \hat{C}\left(\frac{z}{2}, u\right)+(1-\rho) u \hat{C}^{2}\left(\frac{z}{2}, u\right)+(1-u)(1+z) e^{-z} .
$$

7. The Average Cost. Let $C_{1}(z)$ be the exponential generating function of the expected values of $C_{n}$ and let $\hat{C}_{1}(z)=e^{-z} C_{1}(z)$ be its Poisson transform.

Hence, if $\hat{C}_{n}=n !\left[z^{n}\right] \hat{C}_{1}(z)$, then

$$
\mathbb{E}\left[C_{n}\right]=n !\left[z^{n}\right] C_{1}(z)=\sum_{k \geq 0}\left(\begin{array}{l}
n \\
k
\end{array}\right) \hat{C}_{k}
$$

Our plan to get $\mathbb{E}\left[C_{n}\right]$ is therefore more or less standard: (1) from the functional equation satisfied by $\hat{C}_{1}(z)$ it is almost straightforward to obtain its coefficients $\hat{C}_{n}$; (2) an asymptotic estimate for the expected values $\mathbb{E}\left[C_{n}\right]$ sought then follows from the application of Rice's method [8] to (10). 
Proposition 8. The generating function $\hat{C}_{1}(z)$ satisfies

$$
\hat{C}_{1}(z)=1-(1+z) e^{-z}+(2-\rho) \hat{C}_{1}\left(\frac{z}{2}\right) .
$$

Furthermore, its coefficients are, for $n \geq 1$,

$$
\hat{C}_{n}=\frac{(-1)^{n}(n-1)}{1-(2-\rho) 2^{-n}}
$$

and $\hat{C}_{0}=0$.

PROOF. The functional equation satisfied by $\hat{C}_{1}(z)=e^{-z} C_{1}(z)$ trivially follows by differentiating the functional equation given in Proposition 7 with respect to $u$ and setting $u=1$, since

$$
\hat{C}_{1}(z)=e^{-z} C_{1}(z)=e^{-z} C_{u}(z, 1)=\hat{C}_{u}(z, 1) .
$$

From the equation for $\hat{C}_{1}(z)$, we get its coefficients $\hat{C}_{n}$ almost effortlessly:

$$
\hat{C}_{n}=(-1)^{n}(n-1)+(2-\rho) 2^{-n} \hat{C}_{n} .
$$

The proposition above completes the first part of the analysis, so we can now produce an exact solution for the expected value of $C_{n}$, as announced:

$$
\mathbb{E}\left[C_{n}\right]=\sum_{k=1}^{n}\left(\begin{array}{l}
n \\
k
\end{array}\right) \frac{(-1)^{k}(k-1)}{1-(2-\rho) 2^{-k}} .
$$

However, the solution above gives us little information about these expected values. Alternating sums such as the one above are also difficult to evaluate numerically because of the cancellations. Hence, the next step is to produce an asymptotic expansion for $\mathbb{E}\left[C_{n}\right]$, a task which is becoming almost mechanical thanks to increasingly better understood "technologies" such as Rice's method and others akin to the Mellin transform [8]. Briefly stated, we can convert the alternating sum into an integral in the complex plane by a judicious choice of the integration path and then apply the residue theorem:

$$
\begin{aligned}
\sum_{k=n_{0}}^{n}\left(\begin{array}{l}
n \\
k
\end{array}\right)(-1)^{k} f_{k} & =-\frac{1}{2 \pi \mathrm{i}} \int_{\mathcal{C}} f(z) \frac{\Gamma(n+1) \Gamma(-z)}{\Gamma(n+1-z)} d z \\
& =\sum_{\sigma} \operatorname{Res}\left[f(z) \frac{\Gamma(n+1) \Gamma(-z)}{\Gamma(n+1-z)} ; z=\sigma\right],
\end{aligned}
$$

where the last sum extends to all poles $\sigma$ not in $\left[n_{0},+\infty\right)$.

THEOREM 5. Let $C_{n}$ be the cost of a partial matching query with $s$ out of $K$ specified attributes in a random $K d$-trie of size $n$. Then

$$
\mathbb{E}\left[C_{n}\right] \sim \frac{n^{L}}{\log 2}\left((L-1) \Gamma(-L)+\delta_{L}\left(\log _{2} n\right)\right),
$$


where $L=\log _{2}(2-\rho)$ and

$$
\delta_{L}(x)=\sum_{k \neq 0}\left(L-1+\frac{2 \pi \mathrm{i}}{\log 2} k\right) \Gamma\left(-L+\frac{2 \pi \mathrm{i}}{\log 2} k\right) e^{2 \pi \mathrm{i} k x}
$$

is a periodic function of period 1 , mean 0 , and small amplitude $\left(\ll 10^{-6}\right.$, for all $\left.\rho\right)$. Its absolute value is bounded by a small constant depending on $\rho$, slightly larger for larger values of $\rho$; for instance, if $\rho=0.999$ the absolute value is bounded by $5 \cdot 10^{-7}$, for smaller values of $\rho$ the bound is even smaller.

ProOF. Set $f_{k}=(k-1) /\left(1-(2-\rho) 2^{-k}\right)$, so $(11)$ reads

$$
\mathbb{E}\left[C_{n}\right]=\sum_{k=1}^{n}\left(\begin{array}{l}
n \\
k
\end{array}\right)(-1)^{k} f_{k} .
$$

A suitable analytic continuation for $f_{k}$ is obviously

$$
f(z)=\frac{z-1}{1-(2-\rho) 2^{-z}},
$$

whose singularities are located at

$$
z=\chi_{k}=\log _{2}(2-\rho)+\frac{2 \pi \mathrm{i}}{\log 2} k, \quad k=0, \pm 1, \pm 2, \ldots
$$

Each one of these is a simple pole, and, hence,

$$
\sum_{k=1}^{n}\left(\begin{array}{l}
n \\
k
\end{array}\right)(-1)^{k} f_{k}=\sum_{k} n ! \operatorname{Res}\left[f(z) \frac{\Gamma(-z)}{\Gamma(n+1-z)} ; z=\chi_{k}\right] .
$$

Let $L=\log _{2}(2-\rho)$. The residue at $z=\chi_{k}$ is given by

$$
\begin{aligned}
& n ! \operatorname{Res}\left[f(z) \frac{\Gamma(-z)}{\Gamma(n+1-z)} ; z=\chi_{k}\right] \\
& \quad=\frac{L-1}{\log 2}\left(1+\frac{2 \pi \mathrm{i}}{(\log 2)(L-1)} k\right) \cdot n^{L} \cdot e^{2 \pi \mathrm{i} k \log _{2} n} \cdot \Gamma\left(-L-\frac{2 \pi \mathrm{i}}{\log 2} k\right) .
\end{aligned}
$$

The statement of the theorem follows after a few simple manipulations and simplifications.

The behavior of $\delta_{L}(x)$ (its small amplitude and the bounds on absolute values) follows because the modulus of the gamma function decreases extremely fast along any vertical line in the complex plane.

In Figure 5 we plot the difference between $1-\rho$ and the exponent of $n$ in the cost of partial matches for standard $K$ d-trees $(\vartheta(\rho))$, for relaxed $K$ d-tries, and for relaxed $K$ d-trees. The horizontal axis would correspond to standard $K$ d-tries as the exponent in their expected cost is $1-\rho$. The exponent in relaxed (standard) $K$ d-trees is bigger than the exponent in relaxed (standard) $K$ d-tries as expected: tries tend to be more well balanced than search trees. It is a bit more surprising that standard $K$ d-trees perform slightly better than relaxed $K$ d-tries (the difference never exceeds 0.025 , but is not 0 ). The variance due to the relaxation of discriminators is the cause. 


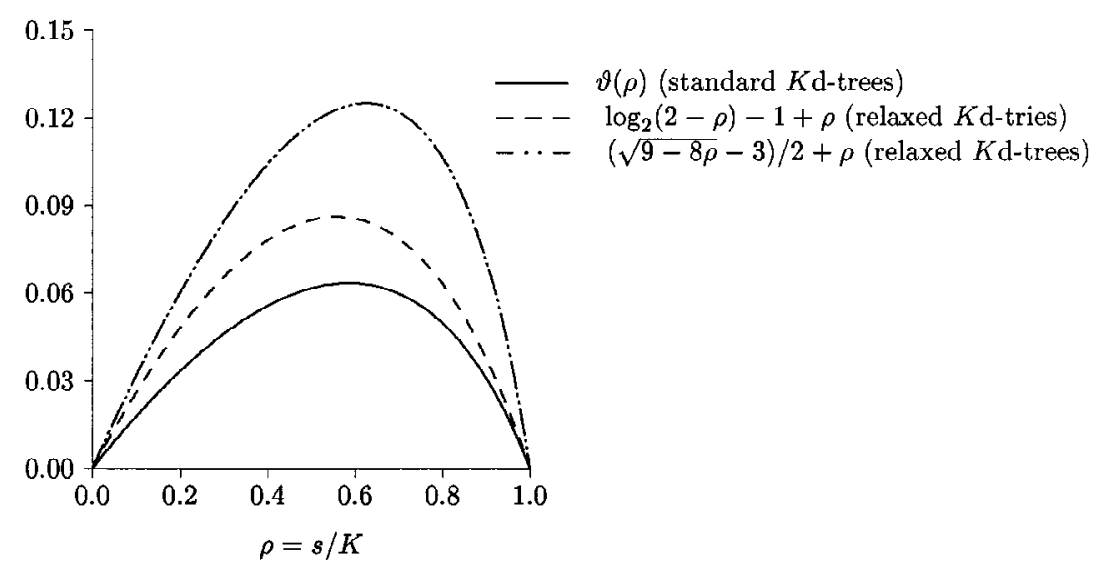

Fig. 5. Excess (with respect to $1-\rho$ ) of the exponent in the average cost of partial match.

8. The Variance. Let $C_{2}(z)$ be the exponential generating function of the second factorial moments of the number of comparisons and let $\hat{C}_{2}(z)$ be its Poisson transform.

PROPOSITION 9. The generating function $\hat{C}_{2}(z)$ satisfies

$$
\hat{C}_{2}(z)=2(2-\rho) \hat{C}_{1}\left(\frac{z}{2}\right)+(2-\rho) \hat{C}_{2}\left(\frac{z}{2}\right)+2(1-\rho)\left(\hat{C}_{1}\left(\frac{z}{2}\right)\right)^{2} .
$$

Furthermore, its coefficients are, for $n \geq 2$,

$$
\begin{aligned}
\hat{C}_{n}^{(2)}= & \frac{(-1)^{n}(n-1)(2-\rho) 2^{1-n}}{\left(1-(2-\rho) 2^{-n}\right)^{2}}+\frac{(-1)^{n}(n-1)\left(2+2^{n-2}(n-4)\right)(1-\rho) 2^{1-n}}{\left(1-(2-\rho)^{2} 2^{-n}\right)\left(1-(2-\rho) 2^{-n}\right)} \\
& +\frac{(-1)^{n}(1-\rho)(2-\rho) 2^{2-n}}{\left(1-(2-\rho)^{2} 2^{-n}\right)\left(1-(2-\rho) 2^{-n}\right)} \\
& \times \sum_{\ell=1}^{n-1}\left(\begin{array}{l}
n \\
\ell
\end{array}\right)(n-\ell-1) 2^{-\ell} \frac{\ell-1}{1-(2-\rho) 2^{-\ell}},
\end{aligned}
$$

and $\hat{C}_{0}^{(2)}=\hat{C}_{1}^{(2)}=0$.

ProOF. From Proposition 7 we get, upon differentiating twice with respect to $u$ and setting $u=1$, the functional equation for $\hat{C}_{2}(z)$.

Reading off the coefficients we find

$$
\begin{aligned}
\hat{C}_{n}^{(2)} & =\frac{n !\left[z^{n}\right]\left[2(2-\rho) \hat{C}_{1}(z / 2)+2(1-\rho)\left(\hat{C}_{1}(z / 2)\right)^{2}\right]}{1-(2-\rho) 2^{-n}} \\
& =\frac{(2-\rho) 2^{1-n}(-1)^{n}(n-1)+(1-\rho) 2^{1-n} n !\left[z^{n}\right] \hat{C}_{1}^{2}(z)}{1-(2-\rho) 2^{-n}} .
\end{aligned}
$$


We thus have to find the coefficients $\hat{A}_{n}=n !\left[z^{n}\right] \hat{C}_{1}^{2}(z)$. From Proposition 8 , squaring the closed form for $\hat{C}_{1}(z)$ we get

$$
\hat{C}_{1}^{2}(z)=\left(1-(1+z) e^{-z}\right)^{2}+2(2-\rho)\left(1-(1+z) e^{-z}\right) \hat{C}_{1}\left(\frac{z}{2}\right)+(2-\rho)^{2} \hat{C}_{1}^{2}\left(\frac{z}{2}\right) .
$$

Therefore, we have

$$
\hat{A}_{n}=\frac{n !\left[z^{n}\right]\left[\left(1-(1+z) e^{-z}\right)^{2}+2(2-\rho)\left(1-(1+z) e^{-z}\right) \hat{C}_{1}(z / 2)\right]}{1-(2-\rho)^{2} 2^{-n}} .
$$

Finally, we obtain the formula sought for the $\hat{A}_{n}$ from

$$
n !\left[z^{n}\right]\left(1-(1+z) e^{-z}\right)^{2}=(-1)^{n}(n-1)\left[2+2^{n-2}(n-4)\right] \quad(n \geq 1)
$$

and

$$
n !\left[z^{n}\right]\left(1-(1+z) e^{-z}\right) \hat{C}_{1}(z / 2)=(-1)^{n} \sum_{k=1}^{n-1}\left(\begin{array}{l}
n \\
k
\end{array}\right)(n-k-1) 2^{-k} \frac{k-1}{1-(2-\rho) 2^{-k}}
$$

for $n \geq 2$. Putting everything together yields the form given in the proposition.

THEOREM 6. Let $C_{n}$ denote the cost of a partial match query with s specified attributes out of $K$ in a random relaxed $K d$-trie of size $n$. Then

$$
\mathbb{V}\left[C_{n}\right] \sim \frac{n^{2 L}}{\log 2}\left(r_{0}(L) \cdot \Gamma(-2 L)-\frac{(L-1)^{2} \Gamma^{2}(-L)}{\log 2}+\delta_{L}^{(2)}\left(\log _{2} n\right)\right),
$$

where $L=\log _{2}(2-\rho)$, and

$$
\begin{aligned}
r_{0}(L)= & \frac{4(2 L-1)}{1-\rho}+(2 L-1)(L-2)(2-\rho) \\
& +4 \sum_{\ell \geq 1}\left(\begin{array}{c}
2 L \\
\ell
\end{array}\right)(2 L-1-\ell) 2^{-\ell} \frac{\ell-1}{1-(2-\rho) 2^{-\ell}} .
\end{aligned}
$$

If $\rho=2-\sqrt{2}$, i.e., $2 L=1$, then $r_{0}(L) \Gamma(-2 L)$ must be interpreted as the corresponding limit when $\rho \rightarrow 2-\sqrt{2}$.

Furthermore, the fluctuating term $\delta_{L}^{(2)}(x)$ has period 1 and small amplitude. The absolute value $\left|\delta_{L}^{(2)}(x)\right|$ is bounded by a constant depending on $\rho$ (actually, on $L=$ $\log _{2}(2-\rho)$; the constant is larger for larger $\left.\rho\right)$. For instance, if $\rho=0.999,\left|\delta_{L}^{(2)}(x)\right|<$ 0.012 . For moderate values and small values of $\rho$, the bound is of the order of $10^{-4}$ or less. The amplitude itself is much smaller, is $\ll 10^{-6}$ for all $\rho$ (see Figure 6 ).

The explicit form of $\delta_{L}^{(2)}(x)$ is

$$
\delta_{L}^{(2)}(x)=\sum_{k \neq 0} r_{k}(L) \cdot \Gamma\left(-2 L+\frac{2 \pi \mathrm{i}}{\log 2} k\right) e^{2 \pi \mathrm{i} k x}-\delta_{L}^{2}(x),
$$




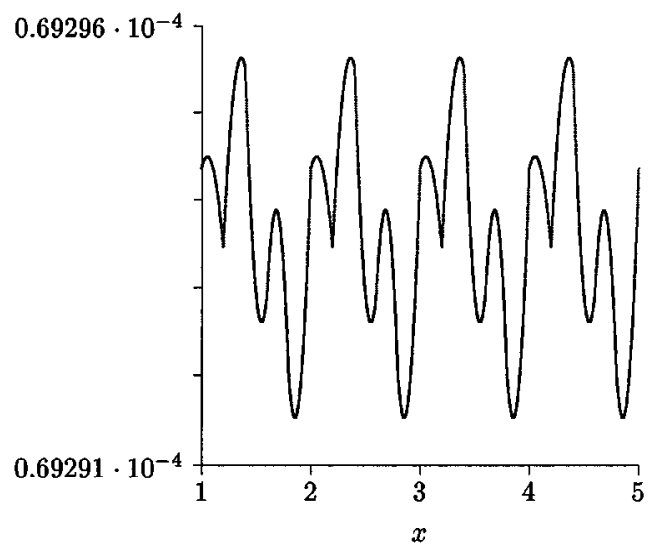

Fig. 6. The fluctuation $\delta_{L}^{(2)}(x)$ in the variance of the cost of partial match in relaxed $K$ d-tries $(\rho=0.5$, $\left.L=\log _{2}\left(\frac{3}{2}\right) \approx 0.585\right)$.

$$
\begin{aligned}
r_{k}(L)= & \frac{4(2 L-1+(2 \pi \mathrm{i} / \log 2) k)}{1-\rho} \\
& +\left(2 L-1+\frac{2 \pi \mathrm{i}}{\log 2} k\right)\left(L-2+\frac{\pi \mathrm{i}}{\log 2} k\right)(2-\rho) \\
& +4 \sum_{\ell \geq 1}\left(\begin{array}{c}
2 L+(2 \pi \mathrm{i} / \log 2) k \\
\ell
\end{array}\right)\left(2 L-1+\frac{2 \pi \mathrm{i}}{\log 2} k-\ell\right) \\
& \times 2^{-\ell} \frac{\ell-1}{1-(2-\rho) 2^{-\ell}}, \quad k= \pm 1, \pm 2, \ldots,
\end{aligned}
$$

and $\delta_{L}(x)$ is as in Theorem 5 .

PROOF. From the closed formula for the second factorial moments $\mathbb{E}\left[C_{n}^{\frac{2}{n}}\right]$ in Proposition 9 , it is relatively easy to obtain the suitable continuation (i.e., $f(k)=\mathbb{E}\left[C_{k}^{\frac{2}{k}}\right]$ for any positive integer $k$ ):

$$
\begin{aligned}
f(z)= & \frac{(2-\rho) 2^{1-z}(z-1)}{\left(1-(2-\rho) 2^{-z}\right)^{2}}+\frac{(z-1)\left(2+2^{z-2}(z-4)\right)(1-\rho) 2^{1-z}}{\left(1-(2-\rho)^{2} 2^{-z}\right)\left(1-(2-\rho) 2^{-z}\right)} \\
& +\frac{(1-\rho) 2^{2-z}(2-\rho)}{\left(1-(2-\rho)^{2} 2^{-z}\right)\left(1-(2-\rho) 2^{-z}\right)} \sum_{\ell \geq 1}\left(\begin{array}{l}
z \\
\ell
\end{array}\right)(z-\ell-1) 2^{-\ell} \frac{\ell-1}{1-(2-\rho) 2^{-\ell}} \\
& +\frac{(1-\rho)(2-\rho) 4^{1-z}(z-1)}{\left(1-(2-\rho)^{2} 2^{-z}\right)\left(1-(2-\rho) 2^{-z}\right)^{2}} .
\end{aligned}
$$

In order to apply Rice's method, we must compute the residues of

$$
\frac{\Gamma(n+1) \Gamma(-z)}{\Gamma(n+1-z)} f(z)
$$


with real part $<2$. First observe that there is no pole at $z=1$, since $f(1)=0$, unless $\rho=2-\sqrt{2}$ (equivalently, $2 L=1$ ). In any case, careful examination shows that the dominant singularities are located at $z=\chi_{k}=2 L+(2 \pi \mathrm{i} / \log 2) k, k=0, \pm 1, \pm 2, \ldots$, and are always simple poles, even if $2 L=1$.

The residue of $f(z)$ at $\chi_{k}$ is

$$
\begin{aligned}
r_{k}^{\prime}(L)= & \frac{4(2 L-1+(2 \pi \mathrm{i} / \log 2) k)}{(1-\rho) \log 2} \\
& +\frac{(2 L-1+(2 \pi \mathrm{i} / \log 2) k)(L-2+(\pi \mathrm{i} / \log 2) k)(2-\rho)}{\log 2} \\
& +\frac{4}{\log 2} \sum_{\ell \geq 1}\left(\begin{array}{c}
2 L+(2 \pi \mathrm{i} / \log 2) k \\
\ell
\end{array}\right)\left(2 L+\frac{2 \pi \mathrm{i}}{\log 2} k-\ell-1\right) \\
& \times 2^{-\ell} \frac{\ell-1}{1-(2-\rho) 2^{-\ell}} .
\end{aligned}
$$

In particular, for $k=0$,

$$
\begin{aligned}
r_{0}^{\prime}(L)= & \frac{4(2 L-1)}{(1-\rho) \log 2}+\frac{(2 L-1)(L-2)(2-\rho)}{\log 2} \\
& +\frac{4}{\log 2} \sum_{\ell \geq 1}\left(\begin{array}{c}
2 L \\
\ell
\end{array}\right)(2 L-\ell-1) 2^{-\ell} \frac{\ell-1}{1-(2-\rho) 2^{-\ell}} .
\end{aligned}
$$

Furthermore,

$$
\frac{\Gamma(n+1) \Gamma\left(-\chi_{k}\right)}{\Gamma\left(n+1-\chi_{k}\right)} \sim \Gamma\left(-\chi_{k}\right) n^{2 L} e^{2 \pi \mathrm{i} k \log _{2} n} .
$$

Factoring out $1 / \log 2$ from the $r_{k}^{\prime}$ 's and collecting together the contributions of the poles with imaginary part as the first term in the fluctuation $\delta_{L}^{(2)}(x)$, we get an asymptotic estimate of $\mathbb{E}\left[C_{n}^{\frac{2}{n}}\right]$ which, after subtracting the square of $\mathbb{E}\left[C_{n}\right]$ (and adding $\mathbb{E}\left[C_{n}\right]$, but this contribution is asymptotically negligible), yields the asymptotic behavior of $\mathbb{V}\left[C_{n}\right]$.

Thus the standard deviation of the cost of partial match in relaxed $K$ d-tries is of the same order of magnitude as its expected value. This time such a behavior is rather surprising as the order of magnitude of the standard deviation of the cost of partial match in all variants of standard multidimensional digital trees is strictly smaller than that of the expected value [12], [18].

9. Other Digital Multidimensional Trees. In [11] the cost of partial match for standard multidimensional digital search trees (DSTs) and Patricia trees (see [14] and [19] for a description of these alternative digital data structures) was analyzed showing that in all cases its expected value is $\Theta\left(n^{1-\rho}\right)$. Also, Kirschenhofer and Prodinger show that, with regard to the constant factor in the main order term of the expected cost, $K$ d-Patricia perform better than $K \mathrm{~d}$-DSTs and, in turn, these perform better than $K$ d-tries. 


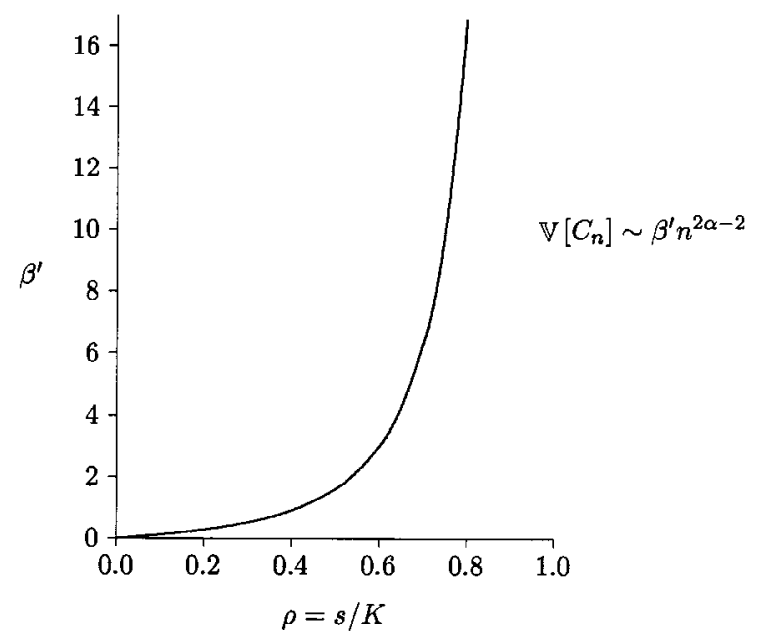

Fig. 7. The value of the constant $\beta^{\prime}$ in the variance of partial match in relaxed $K$ d-tries.

Relaxed variants of $K d$-DSTs and $K$ d-Patricia trees are also easy to define. We do not give the tedious details here, as the techniques that we have to use to analyze partial match in these digital data structures are basically the same that we have used for $K \mathrm{~d}$-tries. For relaxed $K$ d-Patricia trees we have that

$$
\mathbb{E}\left[C_{n}^{[\mathrm{Pat}]}\right] \sim \frac{n^{L}}{\log 2}\left(\Gamma(-L)\left(\frac{\rho}{2} L-\rho\right)+\delta_{L}^{[\mathrm{Pat}]}\left(\log _{2} n\right)\right),
$$

where $L$ is as in Theorem 5 and $\delta_{L}^{[\mathrm{Pat}]}(x)$ has similar characteristics to $\delta_{L}^{[\text {Trie }]}(x) \equiv \delta_{L}(x)$.

For relaxed $K$ d-DSTs we have

$$
\mathbb{E}\left[C_{n}^{[\mathrm{DST}]}\right] \sim \frac{n^{L}}{\log 2}\left(\left(-\frac{Q(2-\rho)}{Q_{\infty}}\right) \Gamma(-L)+\delta_{L}^{[\mathrm{DST}]}\left(\log _{2} n\right)\right),
$$

where

$$
Q(t)=\prod_{n \geq 1}\left(1-\frac{t}{2^{n}}\right), \quad Q_{\infty} \equiv Q(1),
$$

$L$ is as in Theorem 5 , and $\delta_{L}^{[\mathrm{DST}]}(x)$ is yet another fluctuating function that behaves much like $\delta_{L}(x)$.

THEOREM 7. Let $C_{n}^{[\mathrm{Pat}]}, C_{n}^{[\mathrm{DST}]}$, and $C_{n}^{[\text {Trie] }}$ denote the cost of a partial match in a relaxed $K d$-Patricia tree, a relaxed $K d$-DST, and a $K d$-trie, respectively. Then, for any sufficiently large value of $n$,

$$
\mathbb{E}\left[C_{n}^{[\mathrm{Pat}]}\right] \lesssim \mathbb{E}\left[C_{n}^{[\mathrm{DST}]}\right] \lesssim \mathbb{E}\left[C_{n}^{[\text {Trie }]}\right]
$$

PROOF. Comparing Theorem 5 and the expressions given above for the expected costs of partial match in relaxed Patricia and relaxed DSTs, and disregarding the contributions 
of the fluctuating factors, the inequalities follow from (observe that $\Gamma(-L)$ is negative for all $0<\rho<1$ )

$$
\rho-\frac{\rho}{2} \log _{2}(2-\rho)>\frac{Q(2-\rho)}{Q_{\infty}}>1-\log _{2}(2-\rho),
$$

which holds for any $\rho, 0<\rho<1$. This can be proved in a direct manner; however, and surprisingly, it is sufficient to set $y=1-\log _{2}(2-\rho)$; then (12) reads

$$
\left(1-2^{-y}\right)(1+y)>\frac{Q\left(2^{1-y}\right)}{Q_{\infty}}>y,
$$

and those are exactly the inequalities from [11].

10. Final Remarks. The analysis of partial match in relaxed multidimensional trees (either $K$ d-trees or $K$ d-tries) can be done using the same type of techniques that were used to analyze various relevant parameters in other multidimensional data structures, namely, standard $K$ d-trees, standard $K$ d-tries, and $K$-dimensional quadtrees. However, the analysis of relaxed multidimensional trees is usually simpler due to the nice recursive probability models that apply for relaxed trees. The analysis shown in this paper also illustrates that a commonly encountered mistake leads to wrong conclusions: in general it is not valid to assume that the expected value of $f(X)$ is about the same as applying $f$ to the expected value of $X$. In the terms of this paper, even though each possible coordinate is found $t / K$ times-on the average - while following any search path of length $t$ in a relaxed $K$ d-tree, the average performance of partial match in $K$ d-trees is not proportional to that in standard $K$ d-trees (this also holds for relaxed $K$ d-tries versus standard $K$ d-tries).

Nevertheless, the results of this paper also show that there are contexts where using relaxed variants of the multidimensional trees might be useful, as the differences in performance with other multidimensional data structures are not extremely huge (for practical values of $n$ ) and the relaxed trees offer several advantages for the designer of algorithms.

Last but not least, we would like to mention that relaxed $K$ d-trees and relaxed $K$ d-tries can be readily analyzed, at least in a first approximation, with the so-called continuous master theorem [16]. This theorem provides almost effortlessly the right order of magnitude $\left(\alpha(\rho)-1, \log _{2}(2-\rho)\right)$ of the expected cost of partial matches in both relaxed $K$ d-trees and relaxed $K$ d-tries. Unfortunately, it cannot provide information about the constants (constant + fluctuation, in the case of $K \mathrm{~d}$-tries) nor about the order of magnitude of the lower-order terms. Another interesting point is that, using precise asymptotic estimates for the expected values obtained by some other means, the continuous master theorem can be applied to get good estimates of the variances, that is, it produces answers of the form $\beta n^{\varepsilon}+\Theta\left(n^{\varepsilon^{\prime}}\right)$ for the variance of relaxed $K$ d-trees, two-dimensional quadtrees, and relaxed $K$ d-tries, with closed forms for $\beta, \varepsilon$, and $\varepsilon^{\prime}$ in each case, which coincide with those given in Theorems 3,4 , and 6 . 


\section{References}

[1] J. L. Bentley. Multidimensional binary search trees used for associative retrieval. Communications of the ACM, 18(9):509-517, 1975.

[2] W. A. Burkhard. Hashing and trie algorithms for partial match retrieval. ACM Transactions on Database Systems, 1(2):175-187, 1976.

[3] W. Cunto, G. Lau, and P. Flajolet. Analysis of $k \mathrm{~d} t$-trees: $k$ d-trees improved by local reorganisations. In F. Dehne, J.-R. Sack, and N. Santoro, editors, Algorithms and Data Structures (WADS '89), volume 382 of LNCS, pages 24-38. Springer-Verlag, Berlin, 1989.

[4] A. Duch, V. Estivill-Castro, and C. Martínez. Randomized $k$-dimensional binary search trees. In K.-Y. Chwa and O. H. Ibarra, editors, Algorithms and Computation (ISAAC '98), volume 1533 of LNCS, pages 199-208. Springer-Verlag, Berlin, 1998.

[5] P. Flajolet, G. Gonnet, C. Puech, and J. M. Robson. Analytic variations on quadtrees. Algorithmica, 10:473-500, 1993.

[6] P. Flajolet and A. Odlyzko. Singularity analysis of generating functions. SIAM Journal on Discrete Mathematics, 3(1):216-240, 1990.

[7] P. Flajolet and C. Puech. Partial match retrieval of multidimensional data. Journal of the ACM, 33(2):371407, 1986.

[8] P. Flajolet and R. Sedgewick. Mellin transforms and asymptotics: finite differences and Rice's integrals. Theoretical Computer Science, 144(1-2):101-124, 1995.

[9] J. H. Friedman, J. L. Bentley, and R. A. Finkel. An algorithm for finding best matches in logarithmic expected time. ACM Transactions on Mathematical Software, 3(3):209-226, 1977.

[10] R. L. Graham, D. E. Knuth, and O. Patashnik. Concrete Mathematics, 2nd edition. Addison-Wesley, Reading, MA, 1994.

[11] P. Kirschenhofer and H. Prodinger. Multidimensional digital searching — alternative data structures. Random Structures \& Algorithms, 5(1):123-134, 1994.

[12] P. Kirschenhofer, H. Prodinger, and W. Szpankowski. Multidimensional digital searching and some new parameters in tries. International Journal of Foundations of Computer Science, 4:69-84, 1993.

[13] D. E. Knuth. The Art of Computer Programming: Fundamental Algorithms, volume 1, 3rd edition. Addison-Wesley, Reading, MA, 1997.

[14] D. E. Knuth. The Art of Computer Programming: Sorting and Searching, volume 3, 2nd edition. AddisonWesley, Reading, MA, 1998.

[15] R. L. Rivest. Partial-match retrieval algorithms. SIAM Journal on Computing, 5(1):19-50, 1976.

[16] S. Roura. An improved master theorem for divide an conquer recurrences. In P. Degano, R. Gorrieri, and A. Marchetti-Spaccamela, editors, Automata, Languages and Programming (ICALP '97), volume 1256 of LNCS, pages 449-459. Springer-Verlag, Berlin, 1997.

[17] H. Samet. The Design and Analysis of Spatial Data Structures. Addison-Wesley, Reading, MA, 1990.

[18] W. Schachinger. The variance of a partial match retrieval in a multidimensional symmetric trie. Random Structures \& Algorithms, 7(1):81-95, 1995.

[19] R. Sedgewick. Algorithms in C, volume 1, 3rd edition. Addison-Wesley, Reading, MA, 1997.

[20] R. Sedgewick and P. Flajolet. An Introduction to the Analysis of Algorithms. Addison-Wesley, Reading, MA, 1996.

[21] E. T. Whittaker and G. N. Watson. A Course of Modern Analysis. Cambridge University Press, Cambridge, 1952. 